\title{
An Energy-Based Approach for the Multi-Rate Control of a Manipulator on an Actuated Base
}

\author{
Marco De Stefano ${ }^{1,2}$, Ribin Balachandran ${ }^{1}$, Alessandro M. Giordano ${ }^{3}$, Christian Ott $^{1}$ and Cristian Secchi ${ }^{2}$
}

\begin{abstract}
In this paper we address the problem of controlling a robotic system mounted on an actuated floating base for space applications. In particular, we investigate the stability issues due to the low rate of the base control unit. We propose a passivity-based stabilizing controller based on the time domain passivity approach. The controller uses a variable damper regulated by a designed energy observer. The effectiveness of the proposed strategy is validated on a basemanipulator multibody simulation.
\end{abstract}

\section{INTRODUCTION}

Robotic controllers are usually designed to run on a single control unit with a high-enough rate to disregard the effects of discretization. Unfortunately, there are many applications for which this assumption is not valid anymore. This is the case of robotic systems composed of multiple parts that are physically coupled. In these cases, each part is controlled by its own control unit that runs at its own control rate. Some humanoid robots belong to this class of systems [1].

In several space applications, high manipulation capabilities are becoming necessary and a possible solution is given by a manipulator mounted on an actuated floating base (see Fig. 1). The control of the floating base (satellite) enables coarse positioning while the control of the manipulator enables fine dexterous control for complex tasks (e.g. debris recovery). Typically, the control system of the floating base runs at a low rate (between $1 \mathrm{~Hz}$ and $10 \mathrm{~Hz}$ ) while the controller of the manipulator runs at a much higher rate (usually $1000 \mathrm{~Hz}$ ), since the latter has to interact with the environment [2]. Thus, the overall system is composed by two physically coupled mechanical systems with one controlled by a low-rate and the second by a high-rate loop. This mix of frequencies, the presence of zero order holds and possible delays in the communication between the control units can jeopardize the stability of the overall system if not properly taken into account.

Discretization, quantization and delay effects have been investigated in the field of haptics and teleoperation [3], [4]. Effects of discretization were analyzed in [5] for reproducing satellite dynamics on a robotic simulator. An optimal solution for coping with destabilizing effects of time delay has been proposed in [6]. In [1] the wave variables concept was successfully implemented for coordinated control of

\footnotetext{
1 The authors are with the Institute of Robotics and Mechatronics, German Aerospace Center (DLR), 82234 Wessling, Germany.

2 The authors are with the University of Modena and Reggio Emilia, 41100 Modena, Italy.

${ }^{3}$ The authors are with the Department of Informatics, Technical University of Munich (TUM), Garching, 85748, Germany

Contact: marco.destefano@dlr.de
}

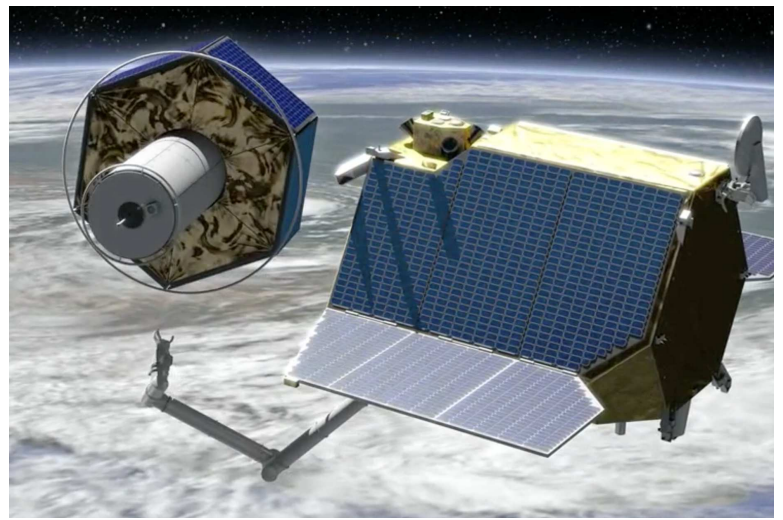

Fig. 1: Space scenario: a controlled spacecraft equipped with a manipulator during a satellite recovery-task.

robots but considering only time delay. The interaction between satellite control and manipulator control of a vehiclemounted manipulator has been first studied in the context of the Shuttle Remote Manipulator [7]. A computed momentum strategy for the coordinated control of reaction wheels and manipulator has been proposed in [8]. A centralized control strategy for vehicle attitude, vehicle position and arm endtip control has been studied in [9], resolving the redundancy of the whole system at the velocity level. At torque level, a centralized control strategy has been proposed in [10], based on a transposed Jacobian algorithm. A resolved-rate control problem was proposed in [11], however the base was not actuated. To avoid fuel consumption, a free-floating strategy has been derived in [12] based on a transposed Jacobian algorithm, assuming no contacts and zero momentum. The problem of the impedance control of a free-floating robot was treated in [13] using feedback linearization. More recently, the transposed Jacobian free-floating strategy has been extended to the nonzero momentum case [14], adding a disturbance compensation term. In [15], the transposed Jacobian free-floating control is extended to stabilize the system even in presence of contacts. This is achieved using actuators only to damp any accumulated momentum in the system. None of the actuated-base strategies [7], [8], [9], [10], [15] addressed the problem of multi-rate control.

In this paper we address the multi-rate control problem of a manipulator on an actuated floating base system from an energy-based perspective. We develop a passivity-based control strategy that guarantees the stabilization of the system independently of the different rates of the controllers. We consider the scenario represented in Fig. 1, where the floating 
base controller runs at $2 \mathrm{~Hz}$, and the manipulator's control unit runs at $1000 \mathrm{~Hz}$. We will show that the presence of multiple control rates leads to a loss of passivity and then we will re-establish a passive behavior and, as a consequence, stability, exploiting the Time Domain Passivity Approach (TDPA) [16].

The contribution of the paper is twofold. First, we identify the energy that can render the system unstable using a network representation. Second, exploiting TDPA [16] we recover a proper energetic behavior and, consequently, stability using a variable control input.

The paper is organized as follows: Sec. II introduces the space robot control and the classical control in continuoustime with the problem statement due to different rate control. The new strategy is presented in Sec. III and proved by simulations in Sec. IV. Conclusions and future works can be found in Sec. V.

\section{SPACE ROBOt CONTROL AND PROBLEM STATEMENT}

In this section, the model of a manipulator on a floating base is introduced. We present the controllers acting on the base and the manipulator. The classical controller results to be stable in continuous time, but when controllers act with different rates, the system may become unstable.

\section{A. Space robot dynamics}

Let us consider a robot with $n$-joints. The general equations of motion for a robot mounted on a moving base [17], are defined as:

$$
\left[\begin{array}{cc}
\mathbf{H}_{b} & \mathbf{H}_{b m} \\
\mathbf{H}_{b m}^{T} & \mathbf{H}_{m}
\end{array}\right]\left[\begin{array}{c}
\ddot{\mathbf{x}}_{\mathbf{b}} \\
\ddot{\mathbf{q}}
\end{array}\right]+\left[\begin{array}{c}
\mathbf{c}_{b} \\
\mathbf{c}_{m}
\end{array}\right]=\left[\begin{array}{c}
\mathbf{F}_{b} \\
\boldsymbol{\tau}
\end{array}\right],
$$

where $\mathbf{H}_{b} \in \mathbb{R}^{6 \times 6}, \mathbf{H}_{m} \in \mathbb{R}^{n \times n}, \mathbf{H}_{b m} \in \mathbb{R}^{6 \times n}$ are the inertia matrices of the whole system, manipulator and the coupling between the base and the manipulator, respectively. The vectors $\ddot{\mathbf{x}}_{\mathbf{b}} \in \mathbb{R}^{6}$ and $\ddot{\mathbf{q}} \in \mathbb{R}^{n}$ are the acceleration of the base (linear and angular) and the acceleration of the robot joints; $\mathbf{c}_{b} \in \mathbb{R}^{6}$ and $\mathbf{c}_{m} \in \mathbb{R}^{n}$ are the non-linear Coriolis/centrifugal terms of the base and of the manipulator, respectively. $\mathbf{F}_{b} \in$ $\mathbb{R}^{6}$ is the force-torque wrench acting on the center of mass of the base-body and $\tau \in \mathbb{R}^{n}$ is the input torque vector.

The end-effector body velocity $\dot{\mathbf{x}}_{\mathbf{m}} \in \mathbb{R}^{6}$ is given by:

$$
\dot{\mathbf{x}}_{\mathbf{m}}=\mathbf{J}_{\mathbf{b}} \dot{\mathbf{x}}_{\mathbf{b}}+\mathbf{J}_{\mathbf{m}} \dot{\mathbf{q}}
$$

where $\mathbf{J}_{b} \in \mathbb{R}^{6 \times 6}$ and $\mathbf{J}_{m} \in \mathbb{R}^{6 \times n}$ are the Jacobian matrices of the base and manipulator, respectively.

The goal is to control the base of the satellite and the manipulator in the inertial frame. We use a quaternion representation in order to define the rotational error. We define $\mathbf{R}_{\mathbf{m}} \in \mathbb{R}^{3 \times 3}$ to be the rotational matrix of the end effector with respect to the inertial frame and $\mathbf{R}_{\mathbf{d}} \in \mathbb{R}^{3 \times 3}$ to be the desired rotational matrix, expressed in the same frame. The error matrix is then defined as $\mathbf{R}_{\phi}=\mathbf{R}_{\mathbf{d}} \mathbf{R}_{\mathbf{m}}^{\mathbf{T}}$. By using the quaternion representation, a scalar $\eta$ and a vector $\hat{\boldsymbol{\varepsilon}} \in \mathbb{R}^{3}$ can be defined, such that the orientation error $\Delta \phi \in \mathbb{R}^{3}$ is then defined as:

$$
\Delta \boldsymbol{\phi}_{m}=2 \mathbf{E}^{\mathbf{T}} \hat{\boldsymbol{\varepsilon}}
$$

where $\mathbf{E} \in \mathbb{R}^{3 \times 3}$ is defined as $\mathbf{E}=I_{3} \eta-\tilde{\boldsymbol{\varepsilon}}$ and where $\tilde{\boldsymbol{\varepsilon}}$ is the known skew-symmetric matrix of the vector $\hat{\boldsymbol{\varepsilon}}$. Analogously, for the position error:

$$
\Delta \mathbf{p}_{\mathbf{m}}=\mathbf{p}_{\mathbf{d}_{\mathbf{m}}}-\mathbf{p}_{\mathbf{m}}
$$

where $\mathbf{p}_{\mathbf{m}}$ is the position of the end effector in the inertial frame and $\mathbf{p}_{\mathbf{d}_{\mathbf{m}}}$ is the desired position. The error vector in position and orientation can be expressed as the vector $\Delta \mathbf{x} \in$ $\mathbb{R}^{6}$, given by:

$$
\Delta \mathbf{x}_{\mathbf{m}}=\left[\Delta \mathbf{p}_{\mathbf{m}} ; \Delta \boldsymbol{\phi}_{m}\right]
$$

Analogously for the base, the error can be defined using (3) and (4). Therefore the error vector for the base will be:

$$
\Delta \mathbf{x}_{\mathbf{b}}=\left[\Delta \mathbf{p}_{\mathbf{b}} ; \Delta \boldsymbol{\phi}_{b}\right]
$$

This representation will be used in the following subsection in order to design the controllers. We assume that the desired pose (i.e. position and orientation) for the manipulator and the base are constant in the inertial frame.

\section{B. Torque controller for the end-effector and the satellite-} base

We consider a manipulator controlled in torque mode with the simple transposed Jacobian:

$$
\tau=\mathbf{J}_{\mathbf{m}}^{\mathbf{T}} \mathbf{F}_{\mathbf{m}}
$$

where $\mathbf{F}_{m} \in \mathbb{R}^{6}$ is the virtual control force applied at the endeffector.

In this paper we use the coordinated control approach [10] and we report the controller in explicit form. Therefore, the virtual Cartesian force $\mathbf{F}_{\mathbf{m}}$ at the end-effector is modeled like a PD (Proportional Derivative) behavior, defined as:

$$
\mathbf{F}_{\mathbf{m}}=K_{P m} \Delta \mathbf{x}_{\mathbf{m}}-K_{D m} \dot{\mathbf{x}}_{\mathbf{m}}
$$

where $K_{P m}$ and $K_{D m} \in \mathbb{R}^{6 \times 6}$ are positive definite matrices, representing stiffness and damping gains of the manipulator controller.

The satellite-base is controlled by the following control law:

$$
\mathbf{F}_{\mathbf{b}}=K_{P b} \Delta \mathbf{x}_{\mathbf{b}}-K_{D b} \dot{\mathbf{x}}_{\mathbf{b}}+\mathbf{J}_{\mathbf{b}}^{\mathbf{T}} \mathbf{F}_{\mathbf{m}}
$$

where $K_{P b}$ and $K_{D b} \in \mathbb{R}^{6 \times 6}$ are positive definite matrices, representing stiffness and damping gains of the base controller and $\mathbf{J}_{\mathbf{b}}^{\mathbf{T}} \mathbf{F}_{\mathbf{m}}$ is a coupling term between manipulator and base.

Considering a non-redundant manipulator and a nonsingular $\mathbf{J}_{\mathbf{m}}$, it can be proven that

$$
\left[\dot{\mathbf{x}}_{b}, \dot{\mathbf{x}}_{m}, \Delta \mathbf{x}_{\mathbf{b}}, \Delta \mathbf{x}_{\mathbf{m}}\right]=\mathbf{0}
$$

is asymptotically stable using the following energetic argument.

Proposition 1: The equilibrium point (10) of (1) is asymptotically stable using the control laws (8) and (9). 
Proof: Consider the total positive definite energy of the system as a candidate Lyapunov function:

$$
\begin{aligned}
& W=\frac{1}{2}\left[\begin{array}{ll}
\dot{\mathbf{x}}_{b}^{T} & \dot{\mathbf{q}}^{T}
\end{array}\right] \underbrace{\left[\begin{array}{cc}
\mathbf{H}_{b} & \mathbf{H}_{b m} \\
\mathbf{H}_{b m}^{T} & \mathbf{H}_{m}
\end{array}\right]}_{\mathbf{H}}\left[\begin{array}{c}
\dot{\mathbf{x}}_{b} \\
\dot{\mathbf{q}}
\end{array}\right]+\frac{1}{2} \Delta \mathbf{x}_{\mathbf{m}}^{T} K_{P m} \Delta \mathbf{x}_{\mathbf{m}}+ \\
& +\frac{1}{2} \Delta \mathbf{x}_{\mathbf{b}}^{T} K_{P b} \Delta \mathbf{x}_{\mathbf{b}} .
\end{aligned}
$$

By computing the derivative and considering the well-known passivity property of the Euler Lagrange systems:

$$
\frac{1}{2}\left[\begin{array}{ll}
\dot{\mathbf{x}}_{b}^{T} & \dot{\mathbf{q}}^{T}
\end{array}\right] \dot{\mathbf{H}}\left[\begin{array}{c}
\dot{\mathbf{x}}_{b} \\
\dot{\mathbf{q}}
\end{array}\right]-\left[\begin{array}{ll}
\dot{\mathbf{x}}_{b}^{T} & \dot{\mathbf{q}}^{T}
\end{array}\right]\left[\begin{array}{c}
\mathbf{c}_{\mathbf{b}} \\
\mathbf{c}_{\mathbf{m}}
\end{array}\right]=0,
$$

we get:

$$
\dot{W}=\dot{\mathbf{x}}_{b}^{T} \mathbf{F}_{\mathbf{b}}+\dot{\mathbf{q}}^{T} \boldsymbol{\tau}-\dot{\mathbf{x}}_{m}^{T} K_{P m} \Delta \mathbf{x}_{\mathbf{m}}-\dot{\mathbf{x}}_{b}^{T} K_{P b} \Delta \mathbf{x}_{b}
$$

Substituting $\boldsymbol{\tau}$ from (7)-(8), $\mathbf{F}_{\mathbf{b}}$ from (9) into (13) and considering the end-effector velocity as in (2), we get:

$$
\dot{W}=-\dot{\mathbf{x}}_{b}^{T} K_{D b} \dot{\mathbf{x}}_{b}-\dot{\mathbf{x}}_{m}^{T} K_{D m} \dot{\mathbf{x}}_{m} \leq 0 .
$$

Therefore, using standard LaSalle arguments, the statement of the proposition is proven.

Thus, we have that in continuous time the system behaves in a dissipative way. In fact stability is achieved by the control laws (8) and (9). As an example we run a simulation in continuous time (see Fig. 2) where the end-effector and the base of the satellite have an initial error. ${ }^{1}$ It can be seen that the error converges in continuous time.

Notice that although we have a control acting on the base, actuators models are not considered.

\footnotetext{
${ }^{1}$ The orientation error is represented by $\psi, \theta, \phi$ (yaw, pitch and roll) angles in the plots.
}
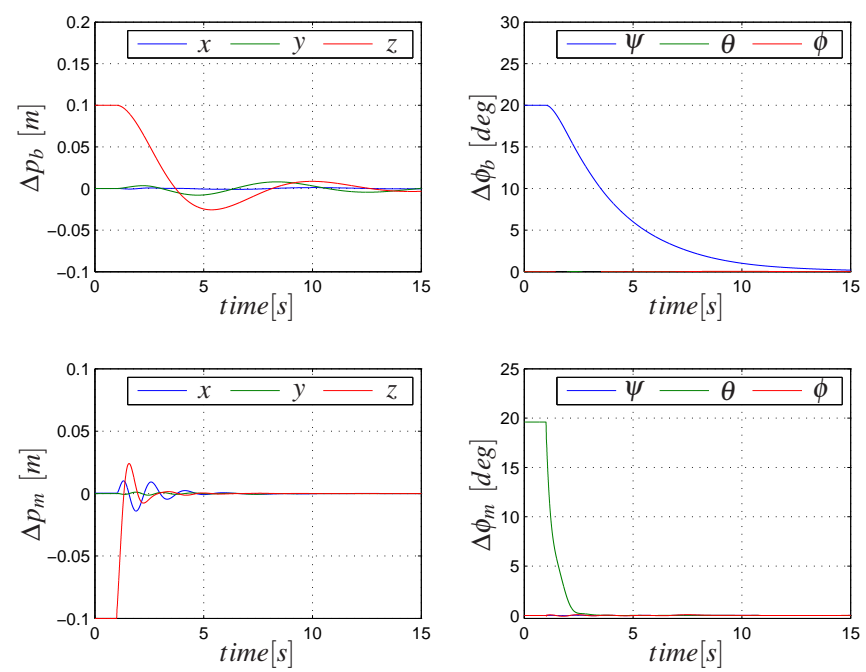

Fig. 2: Stable system with continuous-time controllers: Error of the base in position and orientation (top) and error of the manipulator in position and orientation (bottom).

\section{Problem Statement}

The problem arises when the control laws are computed in discrete-time with different sampling rates. Let $T_{m}$ and $T_{b}$ be the sampling times of the controllers of the manipulator and of the base respectively. We consider the case where the sampling time of the slow rate controller is an integer multiple of the rate of the fast controller, i.e. $T_{b}=n T_{m}$. Therefore at discrete time $k$, we have $k=k_{m} T_{m}=k_{b} T_{b}$ where $k_{m}$ and $k_{b}$ are the discrete steps in each of the controllers. The discrete control laws are given by:

$$
\begin{gathered}
\mathbf{F}_{\mathbf{m}}\left(k_{m}\right)=K_{P m} \Delta \mathbf{x}_{\mathbf{m}}\left(k_{m}\right)-K_{D m} \dot{\mathbf{x}}_{\mathbf{m}}\left(k_{m}\right) \\
\mathbf{F}_{\mathbf{b}}\left(k_{b}\right)=K_{P b} \Delta \mathbf{x}_{\mathbf{b}}\left(k_{b}\right)-K_{D b} \dot{\mathbf{x}}_{\mathbf{b}}\left(k_{b}\right)+\mathbf{J}_{\mathbf{b}}^{\mathbf{T}}\left(k_{b}\right) \mathbf{F}_{\mathbf{m}}\left(k_{b}\right) .
\end{gathered}
$$

It can be proven that the two controllers can interfere due to different sampling rates which may lead to instability. This can be seen with the following example shown in Fig. 3. We consider common control frequencies for the considered scenario which are $T_{m}=0.001 \mathrm{~s}$ and $T_{b}=0.5 \mathrm{~s}$ [2]. We run the simulation considering the discrete laws (15) and (16), with the same conditions of the continuous-case. As it can be seen in Fig. 3, clearly the error in position $\Delta p$ and in orientation $\Delta \phi$ of both controllers diverge. It is worth comparing Fig. 2 (continuous-time control) and Fig. 3 (different rate control) to see the different behaviors of the systems.

This means that the presence of different-rate controllers destroy the dissipative effect shown in Proposition 1 and, therefore, some energy is produced. This implies that the passivity of the system is lost.

In the next section we will design an energy observer in order to identify the energy produced and to dissipate it using a passivity controller.
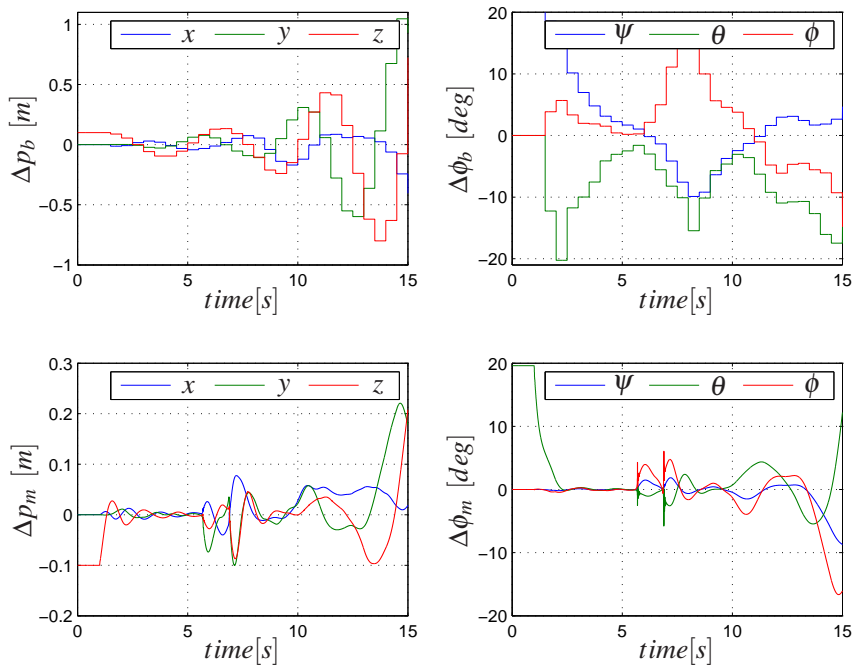

Fig. 3: Unstable system with different-rate controllers: Error of the base in position and orientation (top) and error of the end-effector in position and orientation (bottom). 


\section{ENERGY-BASEd COORdinATED CONTROL}

In this section a port-representation of the mechanical system is proposed in order to perform an energy analysis. The energy produced in the system is identified and it is passivated using a passivity controller. We will exploit the Time Domain Passivity Approach (TDPA) which provides flexibility due to its port-based approach as it does not depend on system modeling [16].

\section{A. Time Domain Passivity}

TDPA is widely used for system stability in practical teleoperation systems which are affected by delays in the communication channel. Delays and packet losses in the communication channel introduce energy in the system and make the system active [18]. An interaction between two processors running at different rates is equivalent to having delays and packet losses between the systems. Therefore, the concept of TDPA is extended in this work to stabilize the system. The underlying principle of TDPA is to observe the input and output energy flows, with a Passivity Observer (PO), of a single-port network, (the virtual environment, in case of haptics) or a 2-port network (the communication channel with delay, in case of teleoperation) [19].

For a one-port system with sample time $T$, the discrete passivity condition is given by:

$$
\sum_{k=0}^{m}\left(\mathbf{F}(k)^{T} \mathbf{v}(k) T\right)+E(0) \geq 0,
$$

where $(\mathbf{F}, \mathbf{v})$ and $E(0)$ are the power correlated variable set and the initial energy storage of the system respectively. If condition (17) holds, the system is passive. The extra energy generated in the port that violates the passivity condition is dissipated with a time-varying damper, the Passivity Controller (PC). This is done in order to ensure that the system is an interconnection of passive ports. This condition will be used to design the controller.

\section{B. Passive coordinated control}

In order to identify the energy introduced into the system by controllers running at different rates, a network representation is derived as shown in Fig. 4. Notice that $S$ is the dynamic system represented by the left hand side of (1), $C_{b}$ is the controller at the base expressed in (16) and $C_{m}$ is the controller of the manipulator (15). The controller $C_{b}$ is represented by electrical elements with impedance $Z_{c b}$ and a dependent voltage source which represents the coupling term of the manipulator, i.e. $J_{b}^{T} F_{m}$. The control rate of the manipulator $C_{m}$ is high and, therefore, its behavior is similar to that of an equivalent system controlled by a springdamper controller (8) in continuous time. This means that the behavior of the controlled manipulator is stable. Thus, the main source of energy is the low rate control which acts on the base (dashed box in Fig. 4).

We observe possible energy injections through the port $\left(\mathbf{F}_{\mathbf{b}}, \dot{\mathbf{x}}_{\mathbf{b}}\right)$. We design an energy observer which runs at the faster rate controller to obtain a greater accuracy. If we render the controlled base passive at this port, then the

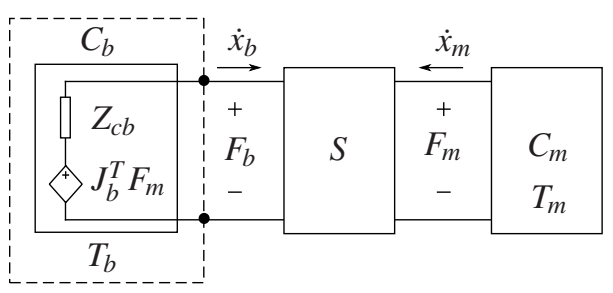

Fig. 4: Network representation of the satellite- manipulator set-up.

overall controlled system will be interconnection of passive systems and, consequently, will be passive [20]. Then all the regenerative and destabilizing effects would be compensated. The PO is designed to observe the energy flowing in and out of this port using (17) with power variables $\left(\mathbf{F}_{\mathbf{b}}, \dot{\mathbf{x}}_{\mathbf{b}}\right)$. In the following description of the TDPA implementation for the multi-dof (degree of freedom) system of the satellite and the manipulator, the PO and PC are applied for each dof separately. It can be mathematically shown that if passivity can be guaranteed for all the dof separately, the overall multidof system is also passive. For a $n$-dof system with initial energy storage $E(0)=0$, the passivity condition for each dof separately leads to:

$$
\sum_{k=0}^{m}\left(\mathbf{F}(k)^{T} \mathbf{v}(k) T\right)=\sum_{k=0}^{m} \sum_{i=1}^{n} F_{i}(k) v_{i}(k) T,
$$

which proves that (17) can be split into a sum of the n-dof components. Therefore, if the passivity condition holds for each component, then the overall system would be passive. We implement the energy observer in the system running at faster rate, as:

$$
\begin{aligned}
E_{o b s}\left(k_{m}\right)=E_{o b s}\left(k_{m}-1\right) & +F_{b}\left(k_{m}\right) \dot{x}_{b}\left(k_{m}\right) T_{m} \\
& +\beta\left(k_{m}-1\right) \dot{x}_{b}\left(k_{m}-1\right)^{2} T_{m} .
\end{aligned}
$$

We assume that the satellite-base velocity $\dot{x}_{b}$ is available with sampling rate $T_{m}$ (eg., using a high-rate position sensor). The energy flowing through the port is updated at each $T_{m}$ and the values $F_{b}$ changes every $T_{b}$. Between two values of $F_{b}$, the observer holds the last received value. Thus, the second term in (19) computes the energy flow of the slow-rate controller. The last term considers the effect of the PC.

The variable damper $\beta$ is derived as:

$$
\beta\left(k_{m}\right)= \begin{cases}-\frac{E_{o b s}\left(k_{m}\right)}{\dot{x}_{b}\left(k_{m}\right)^{2} T_{m}} & E_{\text {obs }}\left(k_{m}\right)<-E(0) \\ 0 & \text { else. }\end{cases}
$$

Notice that the bold notation has been omitted because we are considering the components of the vectors. The impedance correction due to the PC is given by the following quantity:

$$
F_{p c}\left(k_{m}\right)=-\beta\left(k_{m}\right) \dot{x}_{b}\left(k_{m}\right) .
$$

It has to be noted that although the calculation for the PO and PC are implemented at fast rate with a sampling period $T_{m}$, the calculated values of the passivity controller $F_{p c}\left(k_{m}\right)$ are sent to the base controller which runs at $T_{b}$ and it will modify 
the output force $F_{b}\left(k_{b}\right)$. Thus, if the passivity condition is violated (e.g. $E_{o b s}\left(k_{m}\right)<-E(0)$ ), the force commanded to the base will be varied as follows:

$$
F_{b}^{\prime}\left(k_{b}\right)=F_{b}\left(k_{b}\right)+F_{p c}\left(k_{b}\right) .
$$

As a result, the energy will be restored and the observer will be $E_{\text {obs }}(k) \geq-E(0)$ making the network passive. The network representation modified with the passivity controller can be seen in Fig. 5. The benefit of the method is that the control forces depend only on the correlated variables at the port $\left(\mathbf{F}_{\mathbf{b}}, \dot{\mathbf{x}}_{\mathbf{b}}\right)$. Notice that the action of the PC might require high forces at the base and it can be a limitation for the actuator system (e.g. thrusters) which can not fire arbitrarily fast.

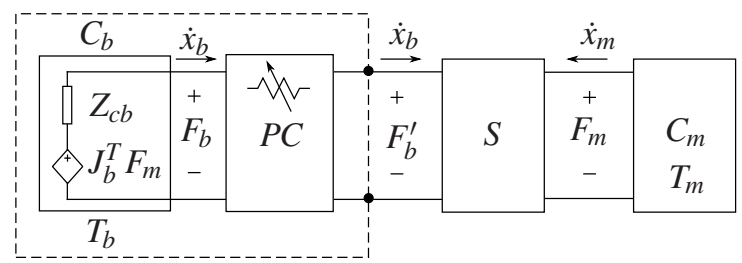

Fig. 5: Network representation scheme modified with PC.

\section{RESUlTS}

This section shows simulation results performed with the proposed method. The simulation environment considers the servicer robot of the OOS-SIM facility [21] able to simulate the dynamics of a free-floating robot on ground, see Fig. 6. The servicer robot is composed of an industrial robot which simulates the dynamics of the satellite and a LWR (Light Weight Robot) mounted at its end-effector. The motion of the satellite-base is related to the dynamic model described in (1). The considered mass of the satellite is $150 \mathrm{~kg}$ with Inertia $I_{x}=38 \mathrm{kgm}^{2}, I_{y}=20 \mathrm{kgm}^{2}, I_{z}=23 \mathrm{kgm}^{2}$. The manipulator arm considered is a 7 dof robot, whose mass and inertia parameters are reported in Table 1.

The simulation runs considering $T_{m}=0.001 \mathrm{~s}$ and $T_{b}=$ $0.5 \mathrm{~s}$. The problem described in Sec. II-C is here resolved by applying the proposed method. Indeed, the destabilizing effects described in Fig. 3 are due to the active observed energy which is shown in Fig. 7 for the linear and angular

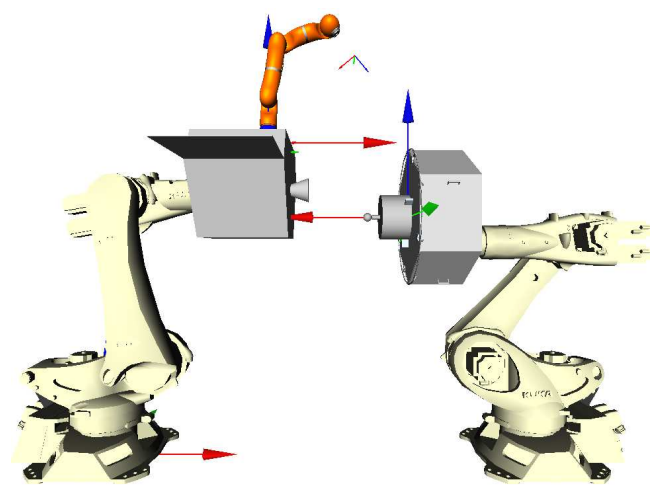

Fig. 6: Simulation environment OOS-SIM.

\begin{tabular}{cccc}
\hline$M m_{\text {link }}[\mathrm{kg}]$ & $I_{x}\left[\mathrm{kgm}^{2}\right]$ & $I_{y}\left[\mathrm{kgm}^{2}\right]$ & $I_{z}\left[\mathrm{kgm}^{2}\right]$ \\
\hline 2.71 & 0.023 & 0.023 & 0.005 \\
2.71 & 0.024 & 0.005 & 0.024 \\
2.54 & 0.013 & 0.013 & 0.005 \\
2.50 & 0.023 & 0.005 & 0.002 \\
1.30 & 0.023 & 0.022 & 0.003 \\
1.57 & 0.003 & 0.003 & 0.003 \\
4.1 & 0.024 & 0.002 & 0.024 \\
\hline
\end{tabular}

TABLE I: Mass and inertia properties of the manipulator arm
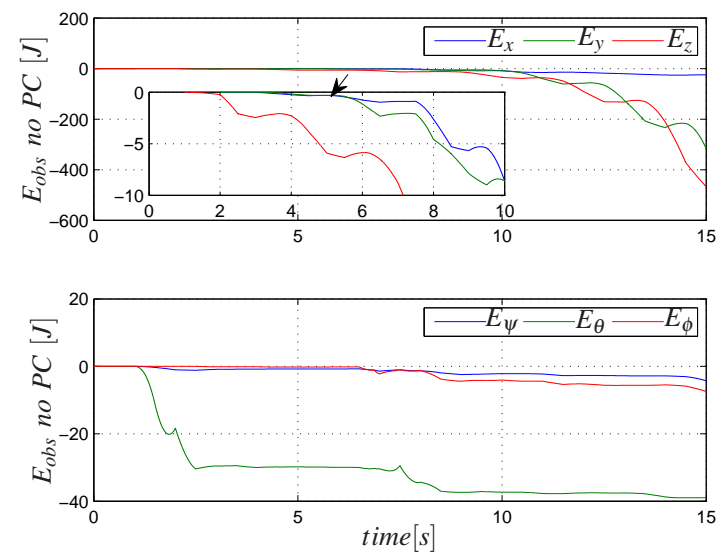

Fig. 7: Energy without PC for translation (top) and rotation (bottom). Active energy is detected by the negative trend.

components. The negative trend of energy shows the activity of the system as the violation of (17) confirms. The proposed approach is then applied. Therefore, the passivity controller will provide the variable force-torque as shown in Fig. 8. This will lead to a passive system as the energy plot shows in Fig. 9. The positive trend indicates that no active energy is pushed into the system. Therefore the error for both systems, i.e. manipulator and base, converges (see Fig. 10) and the system results to be passive. It is worth comparing Fig. 3 (before applying the method) with Fig. 10 (proposed method). The results prove the validity of the method.

Notice that the controller is based on the observability of the port $\left(F_{b}, \dot{x}_{b}\right)$ and it requires the knowledge of $\dot{x}_{b}$ at high rate as discussed in Sec. III.

\section{COnClusions And Future Works}

In this paper, we showed the destabilizing effects of a low-rate control acting on an actuated base which is equipped with a manipulator controlled at high-rate. A network representation has been designed to monitor the energy of the multi-body system and a new control strategy has been proposed. The strategy guarantees passivity thanks to the passivity observer and the passivity controller which dissipates the active energy generated by low-rate control. The method is applied to a multi-body system composed of an actuated space satellite with a robotic manipulator, controlled with different sampling rates. The approach aims at generality so that it can be applied in other domains. Future works aim at extending the approach to the tracking case. 

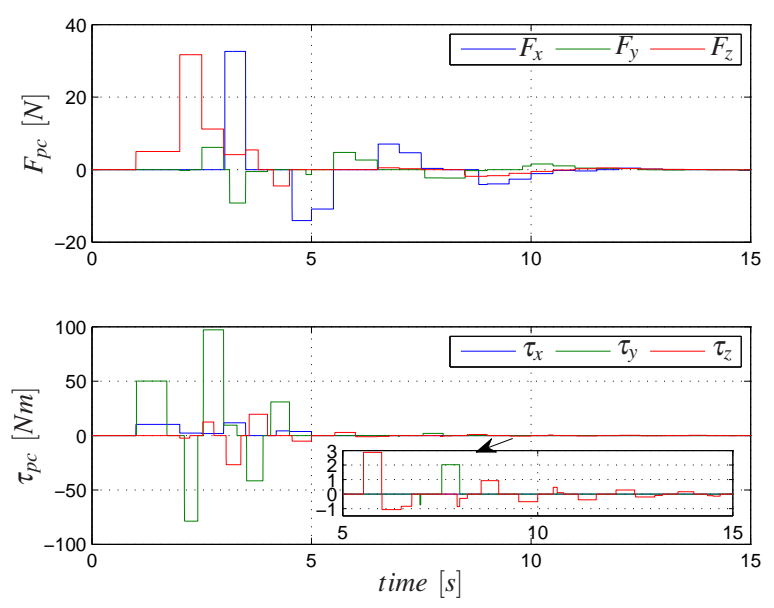

Fig. 8: Force and torque of the passivity controller.
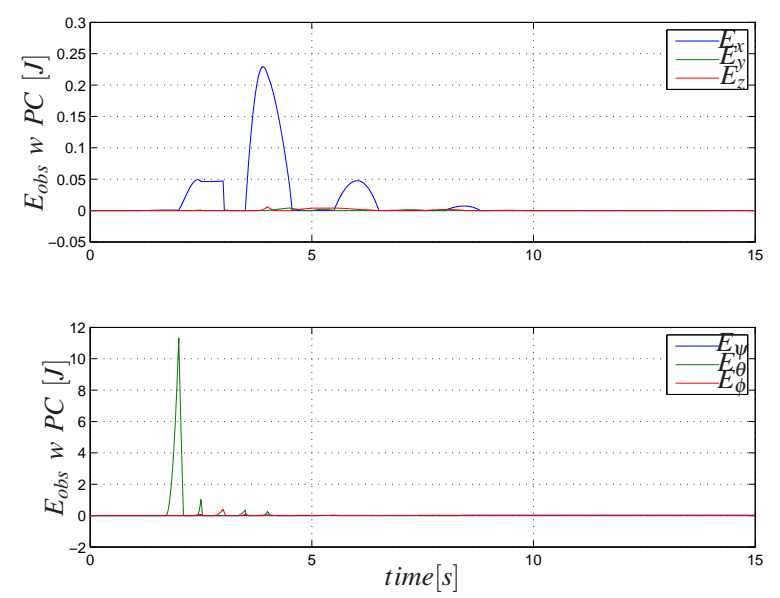

Fig. 9: Energy with PC for traslation (top) and rotation (bottom). Positive energy indicates the passivity of the system.

\section{REFERENCES}

[1] C. Ott and Y. Nakamura, "Employing wave variables for coordinated control of robots with distributed control architecture," in 2008 IEEE International Conference on Robotics and Automation, May 2008, pp. 575-582.

[2] J. Telaar, S. Estable, M. De Stefano, W. Rackl, R. Lampariello, F. Ankersen, and J. Gil Fernandez, "Coupled control of chaser platform and robot arm for the e.deorbit mission," 10th Int. ESA conference on Guidance Navigation and Control Systems (GNC), May 2017.

[3] S. Stramigioli, C. Secchi, A. J. van der Schaft, and C. Fantuzzi, "Sampled data systems passivity and discrete port-hamiltonian systems," IEEE Transactions on Robotics, vol. 21, no. 4, pp. 574-587, Aug 2005.

[4] N. Diolaiti, G. Niemeyer, F. Barbagli, and J. Salisbury, "Stability of haptic rendering: Discretization, quantization, time delay, and coulomb effects," Robotics, IEEE Transactions on, vol. 22, no. 2, pp. 256-268, April 2006.

[5] M. De Stefano, J. Artigas, and C. Secchi, "A passive integration strategy for rendering rotational rigid-body dynamics on a robotic simulator," in 2017 IEEE/RSJ International Conference on Intelligent Robots and Systems (IROS), Sept 2017, pp. 2806-2812.

[6] — - "An optimized passivity-based method for simulating satellite dynamics on a position controlled robot in presence of latencies," in 2016 IEEE/RSJ International Conference on Intelligent Robots and Systems (IROS), Oct 2016, pp. 5419-5426.

[7] R. W. Longman, R. E. Lindbergt, and M. F. Zedd, "Satellite-mounted
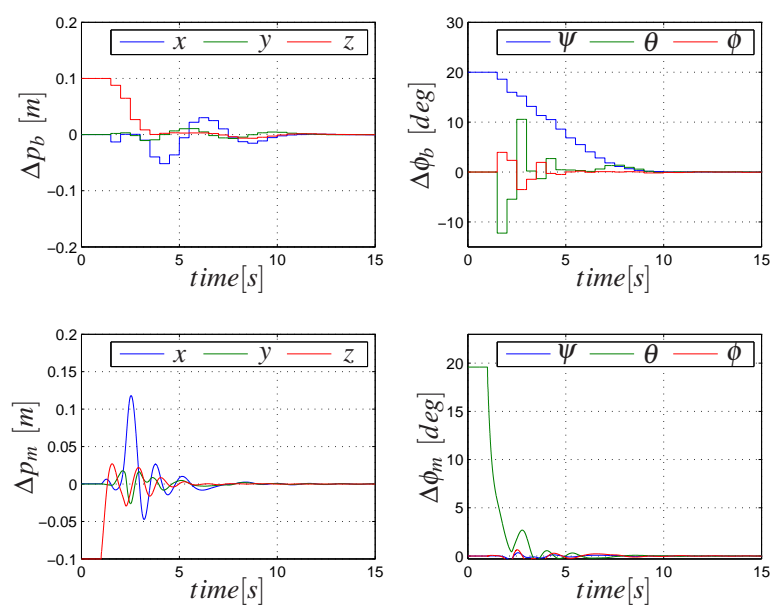

Fig. 10: Stable system with the proposed method. Top row: base error in position and orientation, Bottom row: endeffector error in positon and orientation.

robot manipulators new kinematics and reaction moment compensation," The International Journal of Robotics Research, vol. 6, no. 3, pp. 87-103, 1987.

[8] K. Yoshida, "Practical coordination control between satellite attitude and manipulator reaction dynamics based on computed momentum concept," in IEEE/RSJ/GI International Conference on Intelligent Robots and Systems '94., vol. 3, sep 1994, pp. 1578 -1585.

[9] J. R. Spofford and D. L. Akin, "Redundancy control of a free-flying telerobot," Journal of Guidance, Control, and Dynamics, vol. 13, no. 3 , pp. 515-523, 1990.

[10] E. Papadopoulos and S. Dubowsky, "Coordinated manipulator/spacecraft motion control for space robotic systems," in Proceedings. 1991 IEEE International Conference on Robotics and Automation, Apr 1991, pp. 1696-1701 vol.2.

[11] Y. Umetani and K. Yoshida, "Resolved motion rate control of space manipulators with generalized jacobian matrix," Robotics and Automation, IEEE Transactions on, vol. 5, no. 3, pp. 303-314, Jun 1989.

[12] Y. Masutani, F. Miyazaki, and S. Arimoto, "Sensory feedback control for space manipulators," in Robotics and Automation, IEEE International Conference on, May 1989, pp. 1346-1351 vol.3.

[13] H. Nakanishi and K. Yoshida, "Impedance Control for Free-flying Space Robots -Basic Equations and Applications-," in Intelligent Robots and Systems, IEEE/RSJ International Conference on, Oct 2006.

[14] A. M. Giordano, G. Garofalo, M. De Stefano, C. Ott, and A. AlbuSchffer, "Dynamics and control of a free-floating space robot in presence of nonzero linear and angular momenta," in IEEE 55th Conference on Decision and Control, Dec 2016, pp. 7527-7534.

[15] A. M. Giordano, G. Garofalo, and A. Albu-Schaffer, "Momentum dumping for space robots," in IEEE 56th Conference on Decision and Control (CDC), Dec 2017, pp. 5243-5248.

[16] B. Hannaford and J.-H. Ryu, "Time domain passivity control of haptic interfaces," in Robotics and Automation ICRA, 2001 IEEE International Conference on, vol. 2, 2001, pp. 1863-1869.

[17] R. Featherstone, Rigid Body Dynamics Algorithms. Secaucus, NJ, USA: Springer-Verlag New York, Inc., 2007.

[18] R. Anderson and M. Spong, "Bilateral control of teleoperators with time delay," Automatic Control, IEEE Transactions on, vol. 34, no. 5, pp. 494-501, May 1989.

[19] J.-H. Ryu, D.-S. Kwon, and B. Hannaford, "Stable teleoperation with time-domain passivity control," IEEE Transactions on Robotics and Automation, vol. 20, no. 2, pp. 365-373, 2004.

[20] C. Secchi, S. Stramigioli, and C. Fantuzzi, Control of Interactive Robotic Interfaces: a port-Hamiltonian Approach, ser. Springer Tracts in Advanced Robotics. Springer, 2007.

[21] J. Artigas, M. De Stefano, W. Rackl, R. Lampariello, B. Brunner, W. Bertleff, R. Burger, O. Porges, A. Giordano, C. Borst, and A. AlbuSchaeffer, "The OOS-SIM: An on-ground simulation facility for on-orbit servicing robotic operations," in Robotics and Automation (ICRA), IEEE International Conference on, May 2015, pp. 2854-2860. 